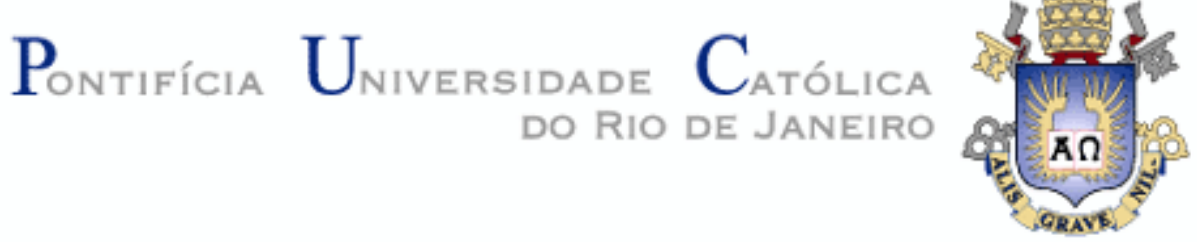

Francine Rossone Silva

\author{
A PAZ LIBERAL NAS \\ OPERAÇÕES DE PEACEBUILDING \\ O 'local' e os limites da crítica
}

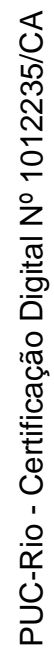

Dissertação de Mestrado

Dissertação apresentada como requisito parcial para obtenção do grau de Mestre pelo Programa de PósGraduação em Relações Internacionais da PUC-Rio.

Orientador: Prof. Kai Michael Kenkel 


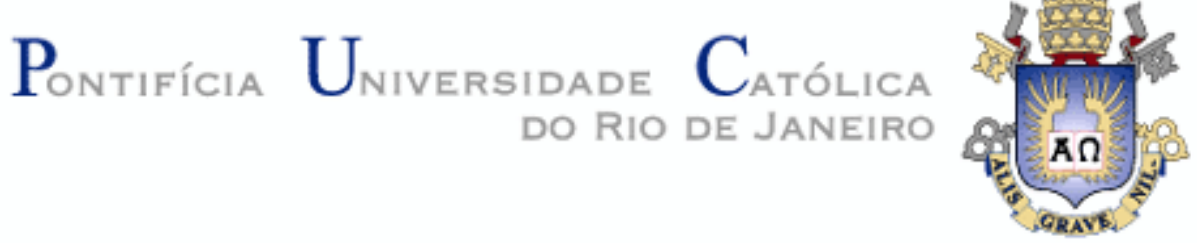

Francine Rossone Silva

\title{
A PAZ LIBERAL NAS \\ OPERAÇÕES DE PEACEBUILDING \\ O 'local' e os limites da crítica
}

\begin{abstract}
Dissertação apresentada como requisito parcial para obtenção do grau de Mestre pelo Programa de PósGraduação em Relações Internacionais da PUC-Rio. Aprovada pela Comissão Examinadora abaixo assinada.
\end{abstract}

Prof. Kai Michael Kenkel

Orientador

Instituto de Relações Internacionais - PUC-Rio

Profa Marta Fernández Moreno

Instituto de Relações Internacionais - PUC-Rio

Prof. Antonio Jorge Ramalho da Rocha Instituto de Relações Internacionais - UnB

Profa Mônica Herz

Coordenadora Setorial de Pós-Graduação e Pesquisa do Centro de Ciências Sociais - PUC-Rio

Rio de Janeiro, 26 de Abril de 2012 
Todos os direitos reservados. É proibida a reprodução total ou parcial do trabalho sem autorização da universidade, da autora e do orientador.

\section{Francine Rossone Silva}

Graduou-se em Relações Internacionais pela Pontifícia Universidade Católica do Rio de Janeiro em 2008.

Ficha Catalográfica

Silva, Francine Rossone

A paz liberal nas operações de peacebuilding: o "local" e os limites da crítica / Francine Rossone Silva; orientador: Kai Michael Kenkel. - 2012.

98 f. ; $30 \mathrm{~cm}$

Dissertação (mestrado) - Pontifícia Universidade Católica do Rio de Janeiro, Instituto de Relações Internacionais, 2012.

Inclui bibliografia

1. Relações internacionais - Teses. 2. Paz liberal. 3. Operações de peacebuilding. 4. Crítica. 5. Paz híbrida. 6. Cultura. I. Kenkel, Kai Michael. II. Pontifícia Universidade Católica do Rio de Janeiro. Instituto de Relações Internacionais. III. Título.

CDD: 327 
Para meus pais, José Maria e Marina 


\section{Agradecimentos}

Ao meu orientador Kai Michael Kenkel, por sua paciência infinita, por suas correções minuciosas e por todo a força e estímulo que tornaram possível a realização e conclusão deste trabalho.

Ao Fundo de Amparo à Pesquisa do Estado do Rio de Janeiro (FAPERJ), pela "Bolsa Nota 10" nos últimos 12 meses de mestrado, e à Pontifícia Universidade Católica do Rio de Janeiro (PUC-Rio).

Aos professores que participaram da Comissão examinadora, pelas colocações enriquecedoras à minha pesquisa.

A todos os professores e funcionários do Instituto de Relações Internacionais (IRI) da PUC-Rio, pelos ensinamentos e pelo apoio.

Aos meus amigos do mestrado, pelas horas de conversas e desabafos.

Ao Leonardo, por me fazer sorrir nos momentos mais atribulados, pelo amor cultivado a cada dia, a cada gesto e a cada palavra de apoio e compreensão.

Aos meus pais, pelo amor e pela vida, por serem meu maior exemplo, minha estrutura e meu porto seguro.

A todos os amigos e familiares que de uma forma ou de outra me estimularam a avançar rumo à conquista dos meus objetivos. 


\section{Resumo}

Silva, Francine Rossone; Kenkel, Kai Michael. A Paz Liberal nas Operações de Peacebuilding: $O$ 'local e os limites da crítica. Rio de Janeiro, 2012. 98p. Dissertação de Mestrado - Departamento de Relações Internacionais, Pontifícia Universidade Católica do Rio de Janeiro.

A dissertação parte do desejo de investigar a proposta da crítica da paz liberal de transcendência da lógica liberal nas operações de peacebuilding. Iniciar essa discussão demanda uma especulação sobre as articulações discursivas que permitiram a construção de uma interpretação de paz em torno de normas e princípios reconhecidos no discurso 'liberal', bem como de noções particulares de institucionalização, democratização e liberalização. A partir desta análise, a pesquisa volta-se para um estudo das narrativas críticas que denunciam a disseminação pelas Nações Unidas de uma receita para a paz baseada em valores hegemônicos e universalizantes, que culmina na construção, por meio das operações de peacebuilding, de uma ordem vazia de significado para a sociedade pós-conflito. Uma vez esclarecido os principais pontos da perspectiva crítica da paz liberal, examina-se a possibilidade de transcendência da paz liberal em prol de uma paz pós-liberal e procura-se demonstrar os paradoxos inerentes à proposta crítica da paz liberal.

\section{Palavras-chave}

Paz liberal; operações de peacebuilding; crítica; paz híbrida; cultura. 


\section{Abstract}

Silva, Francine Rossone; Kenkel, Kai Michael (Advisor). The Liberal Peace in Peacebuilding Operations: The 'local' and the limits of critique. Rio de Janeiro, 2012. 98p. MSc. Dissertation - Instituto de Relações Internacionais, Pontifícia Universidade Católica do Rio de Janeiro.

The dissertation begins with the desire to investigate the proposal of the critique of the liberal peace to transcend the liberal logic in peacebuilding operations. This discussion requires an analysis of the discursive practices that allowed the construction of an interpretation of peace around standards and principles recognized in 'liberal' discourses, as well as particular notions of institutionalization, democratization and liberalization. From this analysis, the research turns to the study of critical narratives that denounces the dissemination by the United Nations of a recipe for peace based on hegemonic and universalizing values, culminating in the construction, through peacebuilding operations, of an order empty of meaning in post-conflict societies. Once clarified the main points of the critical perspective of the liberal peace, it examines the possibility of transcending the liberal peace in favor of a post-liberal peace and seeks to demonstrate the paradoxes inherent to the proposal offered by the critique of the liberal peace.

\section{Keywords}

Liberal peace; peacebuilding operations; critique; hybrid peace; culture. 


\section{Sumário}

1. Introdução 10

1.1 Estrutura da dissertação 14

1.2 A crítica e a emancipação 15

1.3 A crítica, a desconstrução e a genealogia 17

1.4 A crítica e a cultura 18

2. A Paz Liberal nas Operações de Peacebuilding 21

2.1 O liberalismo e o indivíduo 22

2.1.1 O liberalismo e o Estado 27

2.2 Da paz perpétua à paz liberal 30

2.2.1 Algumas interpretações da Paz Perpétua 30

2.2.2 República vs. democracias $\quad 34$

2.2.3 A paz democrática liberal $\quad 35$

2.3 As Nações Unidas e o projeto da paz liberal 40

2.3.1 Transformações das gerações de operações de paz 42

2.3.2 As operações de peacebuilding 44

3. A Crítica da Paz Liberal nas Operações de Peacebuilding 50

3.1 Os problemas, as causas, as soluções 54

3.1.1 Adaptação do 'local' 55

3.1.2 Adaptação ao 'local' 56

3.2 A paz híbrida / pós-liberal nas operações de peacebuilding 59

3.2.1 O problema da paz liberal 60

$\begin{array}{ll}3.2 .2 \text { Hibridização } & 67\end{array}$ 
4. Os Limites da Crítica da Paz Liberal 75

4.1 O hibridismo como resposta à política da identidade 76

4.1.1 O problema ético da paz híbrida $\quad 77$

4.1.2 O paradoxo identidade / diferença 80

4.2 A legitimidade da prática cotidiana 81

4.2.1 Culturas, tradução e violência 83

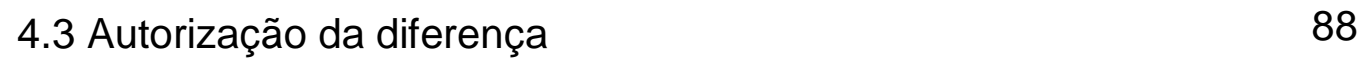

5. Considerações finais 90

6. Referências Bibliográficas 92 